\title{
Rhabdomyolysis recognized after elevation of liver enzymes following prolonged urologic surgery with lateral decubitus position
}

\section{-A case report-}

\author{
Guie Yong Lee, Heeseung Lee, and Youn Jin Kim
}

Department of Anesthesiology and Pain Medicine, School of Medicine, Ewha Womans University Mokdong Hospital, Seoul, Korea

The classic signs and symptoms of rhabdomyolysis are non-specific and not present in all cases, and mild cases might go unrecognized. We present a case of rhabdomyolysis recognized in a 68-year-old man after elevation of liver enzymes following prolonged urologic surgery. The patient's postoperative course was concerned with elevated serum aspartate aminotransferase and alanine aminotransferase without any clinical manifestations on the first postoperative day. After examining the serum creatine kinase and myoglobin levels, the patient was diagnosed with rhabdomyolysis. After 16 days, he was discharged with fully recovered liver enzymes and creatine kinase. We suggest that sufficient perioperative attentions should be given to patients at a higher risk of rhabdomyolysis. (Korean J Anesthesiol 2011; 61:341-343)

Key Words: Lateral decubitus position, Liver enzymes, Rhabdomyolysis.

Rhabdomyolysis is a clinical and laboratory syndrome that is caused by various etiologies. Its intraoperative risk factors include the positioning of a patient during surgery, prolongation of the surgical time and patient obesity [1-6]. Acute renal failure (ARF) is the most serious complication of rhabdomyolysis. However, few reports on liver dysfunction as an associated event of rhabdomyolysis after surgery exist [7]. Here, we report a case of rhabdomyolysis in a patient who had reversible hepatic dysfunction manifested by elevated serum levels of aspartate aminotransferase (AST), alanine aminotransferase (ALT), and lactate dehydrogenase (LD), and decreased albumin that occurred after a prolonged urological procedure in the lateral decubitus position.

\section{Case Report}

A 68-year-old man (height $166 \mathrm{~cm}$, weight $72 \mathrm{~kg}$, BMI 26.1) underwent nephroureterectomy with cuff excision due to a left

Received: October 15, 2010. Revised: 1st, February 11, 2011; 2nd, March 10, 2011; 3rd, March 11, 2011. Accepted: March 11, 2011.

Corresponding author: Guie Yong Lee, M.D., Ph.D., Department of Anesthesiology and Pain Medicine, School of Medicine, Ewha Womans University Mokdong Hospital, 911, Mok-6 dong, Yangcheon-gu, Seoul 158-710, Korea. Tel: 82-2-2650-5040, Fax: 82-2-2655-2924, E-mail: lgyanes@ewha.ac.kr

(ㄷ) This is an open-access article distributed under the terms of the Creative Commons Attribution Non-Commercial License (http:// creativecommons.org/licenses/by-nc/3.0/), which permits unrestricted non-commercial use, distribution, and reproduction in any medium, provided the original work is properly cited. 
ureter carcinoma. The patient had a history of surgery for colon cancer and was on antihypertensive medication. Preoperative physical examination and laboratory investigations showed normal findings.

On arrival in the operating room, his baseline blood pressure was 150/88 $\mathrm{mmHg}$ and his heart rate was 68 beats/minute. After preoxygenation, anesthesia was induced with midazolam, thiopental, and fentanyl. Tracheal intubation was facilitated with rocuronium. Anesthesia was maintained with $\mathrm{N}_{2} \mathrm{O}$, fentanyl and sevoflurane. Surgery was performed for 9 hours in the right lateral decubitus position without repositioning. The patient's blood pressure and heart rate were maintained within a range of 20 percent of baseline during the procedure. Findings from intraoperative arterial blood gas analyses performed every 2 hours were within the normal ranges. The estimated blood loss was $500 \mathrm{ml}$, with no need for a blood transfusion.

After completion of the surgery, the patient was awake and alert. He was then transferred to the postanaesthetic care unit (PACU). In the PACU, intravenous ketolorac was administered for pain relief, and the patient was transferred to the general ward. He complained of mild back pain. Blood chemistry tests that were performed 90 minutes after completion of the surgery revealed AST, $90 \mathrm{IU} / \mathrm{L}$, and ALT, 54 IU/L. The urine volume was maintained at $1.5-3.0 \mathrm{ml} / \mathrm{kg} /$ $\mathrm{h}$ with no darkening of color. Serum levels of AST and ALT increased markedly to $386 \mathrm{IU} / \mathrm{L}$ and $158 \mathrm{IU} / \mathrm{L}$, respectively, on the first postoperative day (day 1) and to $544 \mathrm{IU} / \mathrm{L}$ and $221 \mathrm{IU} /$ $\mathrm{L}$ respectively, on day 2 . Hence, the patient was referred to a gastroenterologist. Since the viral markers were all negative, ischemic liver injury or toxic hepatitis was presumed to have occurred during surgery. However, no hypotensive or hypoxic episodes occurred during surgery. The back pain did not

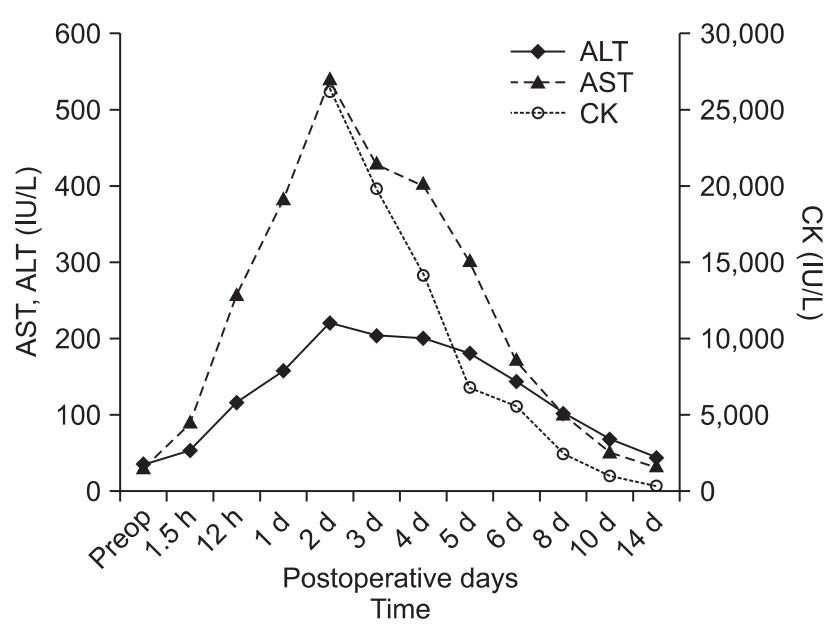

Fig. 1. Serum aspartate aminotransferase (AST), alanine aminotransferase (ALT) and creatine kinase (CK) concentrations during the postoperative period. respond to non-steroidal anti-inflammatory drugs, despite the fact that no tenderness or erythema was present in the thigh or back. Rhabdomyolysis was suspected because the patient had intraoperative risk factors, such as being overweight, having a prolonged operation, and unusual positioning without repositioning. At that time, blood chemistry tests revealed creatine kinase (CK), 26,276 IU/L; serum myoglobin, $\geq 3,000$ $\mathrm{ng} / \mathrm{ml}$ (normal, 5-60 ng/ml); lactate dehydrogenase (LD), 1,002 IU/L and albumin, $3.0 \mathrm{~g} / \mathrm{dl}$. No abnormalities were noted on alkaline phosphatase, prothrombin time, or $\gamma$-GT. Under a diagnosis of rhabdomyolysis, the patient was sufficiently hydrated to maintain high urine output, but alkalinization was not performed because his urine $\mathrm{pH}$ was 7.0. Renal function remained within normal limits during the entire postoperative period. Two weeks later, blood chemistry tests showed CK, 261 IU/L; LD, 239 IU/L; AST, 30 IU/L; and ALT, 42 IU/L. Fig. 1 details the time course of AST, ALT and CK. On day 16, the patient was discharged from the hospital.

\section{Discussion}

Rhabdomyolysis is caused by leakage of muscle enzymes, myoglobin and intracellular constituents from the dissolution of striated muscle fibers. Although rhabdomyolysis can be diagnosed based on a 5 -fold or more increase in the serum CK level [8], recognition of this disease entity is difficult when there are no remarkable clinical symptoms and signs such as gluteal and back pain, reddish brown urine, or oliguria. Our patient's condition went unnoticed until levels of liver enzymes abruptly increased. It is inferred that rhabdomyolysis may have resulted from a direct compressive injury to the striated muscles because the surgery was performed on an overweight patient for 9 hours in the lateral decubitus position without repositioning. Even when the CK level increased to 26,276 IU/L and the myoglobin level increased to $\geq 3,000 \mathrm{ng} / \mathrm{ml}$, the patient's urine volume was well maintained and its color did not change. The classic finding of reddish-brown urine found in rhabdomyolysis was not seen until serum myoglobin reached $100 \mathrm{mg} / \mathrm{dl}$. ARF occurs in $4-33 \%$ of patients. This is considered a prognostic factor in rhabdomyolysis [9]. Serum CK levels correlate with the development of ARF [10]. Fortunately, ARF did not occur in our patient despite a high CK concentration.

The number of reports of rhabdomyolysis associated with reversible liver dysfunction is extremely small. Akmal and Massry [11] reported that reversible hepatic dysfunction appears to occur in $25 \%$ of patients with rhabdomyolysis. Karcher et al. [7] report the case of an obese patient who developed rhabdomyolysis with ARF and hepatic dysfunction. Kim et al. [12] and Park et al. [13] also observed a marked elevation of AST and ALT. The mechanisms for liver dysfunction 
have not yet been elucidated. Horl et al. [14] have demonstrated that proteolytic activity increases in patients with acute renal failure and traumatic rhabdomyolysis. Hepatic dysfunction in rhabdomyolysis may be attributed to liver damage caused by proteases released from injured muscle.

In our patient, drugs which can affect liver function, were not administered during the perioperative period. In addition, no events, such as hypotension or hypoxia during surgery, were present. Serum ALT level reached a peak on day 2, and then decreased together with the serum CK level. Transient liver dysfunction in our patient is believed to have been a concomitant phenomenon related to rhabdomyolysis.

In conclusion, we present a case of rhabdomyolysis with liver dysfunction that occurred after a prolonged urologic procedure in the lateral decubitus position. We would like to emphasize that sufficient attention should be given, during the perioperative period, to patients with a higher risk of rhabdomyolysis.

\section{References}

1. Visweswaran P, Guntupalli J. Rhabdomyolysis. Crit Care Clin 1999; 15: 415-28.

2. Targa L, Droghetti L, Caggese G, Zateli R, Roccella P. Rhabdomyolysis and operating position. Anaesthesia 1991; 46: 141-3.

3. Alterman I, Sidi A, Azamfirei L, Copotoiu S, Ezri T. Rhabdomyolysis: another complication after prolonged surgery. J Clin Anesth 2007;
19: 64-6.

4. Hofmann R, Stoller ML. Endoscopic and open stone surgery in morbidity obese patients. J Urol 1992; 148: 1108-11.

5. Merino I, Borrat X, Balust J, Delgado S, Lacy AM, Vidai J, et al. Rhabdomyolysis after bariatric surgery: a potentially fatal complication. Br J Anaesth 2009; 102: 283-4.

6. Lagandre S, Arnalsteen L, Vallet B, Robin E, Jany T, Onraed B, et al. Predictive factors for rhabdomyolysis after bariatric surgery. Obes Surg 2006; 16: 1365-70.

7. Karcher C, Dieterich HJ, Schroeder TH. Rhabdomyolysis in an obese patient after total knee arthroplasty. Br J Anaesth 2006; 97: 822-4.

8. Lane R, Phillips M. Rhabdomyolysis. BMJ 2003; 327: 115-6.

9. Bagley WH, Yang H, Shah KH. Rhabdomyolysis. Intern Emerg Med 2007; 2: 210-8.

10. de Meijer AR, Fikkers BG, de Keijzer MH, van Engelen BG, Drenth JP. Serum creatine kinase as predictor of clinical course in rhabdomyolysis: a 5-year intensive care survey. Intensive Care Med 2003; 29: 1121-5.

11. Akmal M, Massry SG. Reversible hepatic dysfunction associated with rhabdomyolysis. Am J Nephrol 1990; 10: 49-52.

12. KimTK, Yoon JR, Lee MH. Rhabdomyolysis after laparoscopic radical nephrectomy. Korean J Anesthesiol 2010; 59(Suppl): S41-4.

13. Park SE, Kim DY, Park ES. Hyperkalemia in a patient with rhabdomyolysis and compartment syndrome. Korean J Anesthesiol 2010; 59(Suppl): S37-40.

14. Horl WH, Stepinski J, Schäfer RM, Wanner C, Heidland A. Role of proteases in hypercatabolic patients with renal failure. Kidney Int Suppl 1983; 16: S37-42. 